\title{
Finding the time: Exploring a new perspective on students' perceptions of cosmological time and efforts to improve temporal frameworks in astronomy
}

\author{
Laci Shea Brock \\ Lunar and Planetary Laboratory University of Arizona, 1629 E. University Boulevard, \\ Tucson, Arizona 85721-0092, USA \\ Edward Prather and Chris Impey \\ Steward Observatory University of Arizona, 933 N. Cherry Avenue, Tucson, Arizona 85721-0065, USA
}

(Received 10 May 2017; published 15 June 2018)

\begin{abstract}
[This paper is part of the Focused Collection on Astronomy Education Research.] One goal for a scientifically literate citizenry would be for learners to appreciate when the Earth came to be and where it resides in the Universe. Understanding the Earth's formation in time in both a sociohistorical and scientific sense allows us to place humanity within the larger context of our existence in the Universe. This article considers prior research from cognitive science, psychology, history, and Earth and space science education to inform a new research agenda in astronomy education. While there exists prior research related to learner's ideas of time and the Earth's location, research on how to help students develop a coherent model of the Earth's place in space and time in the Universe is still lacking. We highlight a set of preliminary findings from a pilot study that is part of this new agenda, which is focused on students' ideas on how to connect the Earth's formation with prior events in the Universe.
\end{abstract}

DOI: 10.1103/PhysRevPhysEducRes.14.010138

\section{MOTIVATION FOR RESEARCH}

Understanding time is a cross-disciplinary endeavor uniting the disciplines of astronomy, cosmology, geology, evolutionary biology, and natural history. The ability to comprehend and synthesize temporally related events has been cited as a significant component of scientific and numerical literacy, as well as a critical component of science education standards from kindergarten through the collegiate level. As humans, we are only able to experience and perceive time in short anthropomorphic forms of measurement, such as days or years; however, the perception of cosmic evolutionary time-from the origin of the Universe 13.8 billion years ago to the present day-remains intangible and abstract. Grasping (and accepting) such enormous scales of time as beings who live relatively short lifetimes in comparison is a unique cognitive challenge.

Integrating such macroevolutionary timescales into one's framework is essential in order to learn astronomy. Astronomy can be a challenging discipline to teach and learn-it deals with unimaginably large objects separated

\footnotetext{
*laci@1pl.arizona.edu
}

Published by the American Physical Society under the terms of the Creative Commons Attribution 4.0 International license. Further distribution of this work must maintain attribution to the author(s) and the published article's title, journal citation, and DOI. by vast, untraveled distances that form and evolve on immense timescales much longer than human lifetimes. Researchers have investigated how students of all ages construct their knowledge of astronomical phenomena for nearly a century [1-4]. Recent astronomy education reviews cite students' conceptions of observational topics including objects in the sky, motions within the EarthMoon-Sun system (e.g., lunar phases, eclipses, day-night cycle), gravity, and the cause of seasons are commonly investigated $[4,5]$. Though these aforementioned topics are among the most commonly taught in introductory astronomy courses [6], missing from the literature is how students conceive cosmological and geological time in an astronomical context. Acquiring knowledge of such timescales requires students to develop and apply temporal and spatial reasoning skills, which ultimately shapes a perception of Earth and its place in space and time in the Universe.

Astronomical research continues to demonstrate that Earth is only one of potentially billions of planets in our galaxy alone. Exoplanets - planets in other Solar Systemsare being discovered at a rapid rate, and the question of whether we are alone in the Universe has finally come into focus. This current era of astronomical research has led to the inclusion of new topics within astronomy. Astrobiology-a relatively new interdisciplinary field of science studying the origin, evolution, and distribution of life in the Universehas made its way into both high school and college science classrooms. Implementing astrobiology courses has recently 
been viewed as an ideal way to engage students and adults in science due to the subject's multidisciplinary nature and appeal [7-9]. Our understanding of the origin, formation, and evolution of the Universe has increased drastically over the past few decades as well, and cosmology has become more widely incorporated into existing astronomy courses [10]. Further, more disciplines are including the event that formed our Universe, the big bang, even outside the context of astronomy courses [11].

Incorporating new astronomical topics, such as those previously described, into the classroom are not without challenges, however. Deeper investigations have shown that students often describe the big bang in a manner that is not consistent with the modern cosmological model, instead describing it as a phenomenon organizing preexisting matter (e.g., subatomic particles, molecules, stars, or planets). Students also conflate the big bang with later planetary formation events $[10,12,13]$, which are fundamentally different physical processes.

In this generation in the classroom, students' preinstructional knowledge upon entering introductory astronomy courses may be beginning to shift to incorporate topics that have recently become more commonplace-astrobiology, cosmology, and exoplanets. Students are now faced with a larger pool of information to comprehend when discussing Earth's place in space and time in the Universe in the astronomy classroom. Instructors are equally challenged, tasked with deciding what content to teach and how to most effectively convey the information. This new framing of astronomy establishes a new trajectory for research in science education. Thus, it is of critical importance to investigate how learners are constructing knowledge of Earth's place in time and space to develop effective curricula and instruction in astronomy courses at all levels.

\section{THEORETICAL FRAMEWORK}

Before we investigate any prior research findings, it is important to establish our theoretical framework, which guides this work. This research is informed by a constructivist learning perspective. Constructivism suggests that learners develop their understanding of the physical world through personal experiences, observations, and the integration of new concepts and information [14]. We briefly highlight three facets of constructivism-mental models, phenomenological primitives, and resource framing - that most appropriately guide our agenda.

Collective factors that shape one's representation and understanding of the physical world have been referred to as "mental models" [15-17]. Vosniadou and Brewer [16] explain mental models are generated from and constrained by students' underlying conceptual structures and are used to provide causal explanations of scientific phenomena. As we look toward our research, we will see students constructing mental models influenced by their underlying assumptions of cosmic events prior to Earth's formation.
However, students do not always form coherent representations of Earth nor the events that took place prior to Earth's formation. Students also use their fragmented scientific knowledge to explain astronomical phenomena. DiSessa [18] developed phenomenological primitives, or $p$ prims, as a means to explain how students also rely on more abstract or intuitive knowledge structures. This can be observed when students use the concept of closer means stronger to explain Earth's seasons (i.e., the false concept that Earth is closer to the Sun during summer) [19].

In order for students to consider when Earth formed in relation to the formation of the Universe, they must be able to apply knowledge from multiple disciplines-astronomy, chemistry, physics, and biology, for example-and extrapolate back through cosmological time. Hammer et al. [20] explore how students apply what they know from one scientific context to another based on an activation of resources. Hammer's approach, called resource framing, views learning as a state rather than the acquisition of a "cognitive object." This approach can help students grasp concepts such as Earth's place in space and time in the Universe that cannot be physically experienced.

Throughout this paper we will not interpret research but simply report on it. As we look toward our own research, we will see the aforementioned models of constructivist learning being used. First we will unpack a small portion of research completed related to Earth's place in time and space in the Universe with the purpose of framing the research direction we intend to go. This will ultimately set a new course for research on students' understanding of cosmological time and Earth's formation.

\section{A. The missing link}

At the heart of appreciating Earth's relationship with the Universe is through the concept of time. All physical science disciplines require their learners to possess some grasp of time- be it the time required for an event to happen, or for change to occur [21]. Time is inherent in order to grasp Darwin's theory of evolution, piece together Earth's geological history, approach the concept of climate change, or predict the eventual death of our Sun. Time is a central and fundamental component of describing how a system functions, but its importance stretches far beyond science. Cervato and Frodeman [22] highlight the cultural, economic, religious, and political relevance of time in addressing the looming environmental crises that require a perspective and appreciate of immense timescales. Temporal knowledge has been cited as a significant component of scientific literacy $[23,24]$ and listed in science education standards from K-12 [25-27] through the collegiate level [28]; yet, it is often overlooked in the classroom, or expected to become obvious within the context of the scientific content being taught.

Time as a teaching and learning topic has continued to elude its students and instructors. Available literature 
demonstrates students' conceptual difficulties with time at the elementary $[29,30]$, high school $[31,32]$, and college levels [33-37]. Students' knowledge of geological time has been widely explored and equally so have children's perspectives of Earth [16,38]. However, studies exploring students' understanding of time in an astronomical context are lacking, as well as investigations on more interdisciplinary topics regarding Earth's place in time and space in the Universe [5]. Investigations on students' perceptions of Earth's place in time and space in the Universe may help to break down disciplinary boarders within Earth and space sciences through efforts that consider how both Earth and humanity fit into the cosmic landscape. Students and the general public must develop causal, chronological frameworks that more closely mirror scientific understandings of the relationship between cosmological and geological time-as well as knowledge of Earth's place in the Universe-to become both quantitative and scientifically literate citizens able to deal with global issues of the future.

Studies regarding the temporal knowledge of learners within Earth and space sciences span multiple research areas including cognitive science, psychology, and science education. First, we must unpack a small portion of research done in these areas with the purpose of framing the research direction we intend to go. Our new research agenda incorporates an approach to astronomy education from both the philosophical and literal perspective of what Earth's place in space and time in the Universe is, which undoubtedly requires a grasp of students' knowledge of Earth's age as well as physical location within the Universe. It is through this exploration we can assess how students' knowledge of astronomical content and views of astronomy as a discipline are evolving with increasing scientific discoveries, such as the plethora of exoplanets now known to exist. For clarity, we use the word time or temporal to refer to the essence of time, or more concretely, the existence of both cosmological and geological timescales. The use of the word space or spatial describes both physical location and an awareness of Earth from a spacebased frame of reference.

\section{UNPACKING HOW WE THINK ABOUT EARTH, SPACE, AND TIME}

We first consider research that illustrates how learners in general think about the ideas of time and Earth without applying them to the idea of the Universe. We will then consider research which examines learners' ideas related to how time and Earth are connected to the larger infrastructure of the Universe.

\section{A. Earth as an astronomical body}

Time is a rather abstract concept, especially across large timescales central to the disciplines of Earth and space sciences. While scientists' knowledge of Earth's age and the age of the Universe was first developing, some of the earliest studies by psychologists and cognitive scientists investigated the acquisition of spatially related knowledge in astronomy [1,2]. Researchers first explored children's perspectives of Earth as both a physical and astronomical object $[39,40]$. All people encounter Earth and space science phenomena prior to any formal instructionobserving the Sun, Moon, and stars; experiencing weather, the changing seasons. This leads to initial ideas and beliefs of the larger picture of how Earth fits into the cosmic landscape. Initial studies probing student-constructed knowledge of astronomy helped to shape the constructivist learning movement [14], leading conceptual change researchers to form theoretical approaches to explain how humans learn. Two of these approaches, colloquially known as "knowledge as theory" and "knowledge as elements" or "pieces," explain how learners might progress from naive to scientific understandings of phenomena $[17,18,41,42]$.

Notable work was conducted by Nussbaum and Novak [39] and Vosniadou and Brewer [16,38]. The researchers argued students construct mental models, or theorylike structures, to explain scientific phenomena. Individual mental models spanned a spectrum from scientifically accurate to inaccurate. This approach has been used to examine children's conceptions of Earth's shape, the daynight cycle, and gravity. It is relevant to our work because it enables us to observe how students view Earth as an astronomical body rather than as the planet we live on. It was found that young children's initial perceptions of Earth, its place in the Solar System, and its position in space closely resembled that of Ancient Greek's geocentric perspectives. For example, elementary school-aged children were more likely to conceptualize a flat, unmoving Earth reminiscent of a pre-Aristotelian view [16]. Children aged 10 to 11 years were more likely to acknowledge Earth is both spherical and rotating, suggesting that some scientific explanations of astronomical phenomena had been acquired by this age [16].

A more detailed discussion of work on Earth's shape and related topics-including articles not discussed here-can be found in Bailey and Slater's [4] review. Since the aforementioned review was published, additional work has expanded upon the efforts of Vosniadou and colleagues. Straatemeier et al. [43] investigated how young children in Netherlands constructed mental models of Earth utilizing Vosniadou and Brewer's [16,38] identified mental models and found conflicting results. In their work, Straatemeier and colleagues evaluated a larger sample size of children and concluded children's presuppositions are not strong enough to suggest children actually do construct mental models, rather the children's knowledge is more fragmented and inconsistent [43]. A longitudinal study by Hannust and Kikas [44] used open-ended questions and drawing tasks similar to Vosniadou and Brewer [16] to 
examine two and three year old children's knowledge of Earth over four years. Hannust and Kikas concluded, much like Straatemeier and colleagues, that there were few indications to suggest children did form mental models of Earth at this age. When we examine how learners might perceive Earth's place in time and space in the Universe, it is important for us to consider these aforementioned works that suggest astronomical knowledge appears to be both mental modellike at times and fragmented at others $[16,38,43-45]$.

\section{B. Conceptualizing time}

Time is conceptualized metaphorically, but what is critical for the work we are pursuing is also to consider how time is viewed spatially, which one must do to engage in space in the Universe. The relationship between time, space, and students' subsequent difficulty with grasping these concepts can be observed when students are given tasks that relate time and space, such as interpreting Hubble plots [10]. Prior research further illustrates how learners have a difficult time accounting for motions of the night sky appropriately using Earth as a moving reference frame [46,47]. Our work will ask students to go beyond these concepts by considering information on distance to reason about events in time in the Universe as related to Earth. Students will need to consider events in Earth history from a space-based reference frame, thus we must consider relevant literature on the conceptual understanding of time and time perception from a psychological perspective as well. We follow discussion on time perception with research on time and space in astronomy education.

Researchers in psychology have demonstrated a key link in the conceptual understanding of time through its relationship with spatial thinking $[48,49]$. For example, Levin [50] found that preschool children shape their sense of time by temporally sequencing familiar events into the space of their day, such as snack time or recess time. Cognitive studies show the abstract perception of time is acquired through metaphorical mappings in language from the domain of space; these conceptualizations of time often vary for different cultures and languages. In English, time is commonly perceived through ego-moving or time-moving metaphors. Gentner [42] explains the ego-moving metaphor as a mapping where the observer progresses along a timeline toward the future. The time-moving metaphor is the projection of a "river or conveyor belt on which events are moving from the future to the past" [42]. In other words, time-moving metaphors focus on placing events on a timeline with regards to when the event occurred or will occur, rather than how the event occurred in relation to an individual's location. Most English speakers tend to conceptualize ego-moving metaphors, by placing time on a horizontal axis and expressing they are looking ahead to the future or back into the past [51].
Chrysikou and Ramey's [48] work on time metaphors in language provide some interesting results that may be applicable to time in a scientific context. Participants' responses to ambiguous questions related to the rescheduling of events were evaluated, and the researchers observed biases in responses based on the valence, or intrinsic attractiveness, of the event. Chrysikou and Ramey [48] argued "the perception of time is a complicated interaction of an array of variables" and suggest "subjective factors can modulate one's perception of time ... and may interact with other personality and spatial variables." Similarly, Kurby and Zacks [52] explain how peoplewithout intentionally meaning to-hierarchically break down events to build mental representations and remember what happened, such as separating the act of making a sandwich into discrete events (e.g., collecting ingredients, assembling the sandwich, and clean-up). Therefore, in order to understand the hierarchical structure of an event, one must be able to separate the event into smaller, more meaningful segments [52].

Scientifically speaking, this suggests learners' perceptions of cosmological time or geological time depend not only on scientific content knowledge and quantitative reasoning skills, but also individual feelings and motivations toward the subject, interest in learning it, and concern for its importance in the scale of their personal lives. We have observed behavior representative of these hierarchical arrangements in our pilot research, notably when we asked undergraduate students to respond to an open-ended question and temporally relate the formation of the Universe to the formation of Earth. Students frequently unify these two formation events in their responses, describing that Earth and the Universe came into existence at the same time. Students who did provide numerical estimates and separated the formation events, however, commonly responded "millions or billions of years apart," seemingly unaware of the 3 order of magnitude difference between these numbers. Yet, the students knew in the context of their personal lives millions or billions of years were both large numbers.

Work on time perception also shows a link between temporal and spatial association through the concept of scale that is relevant to our work. For example, recalling past events requires location-based associations to determine how recent an event is, as well as a distance-based association to determine how much time has elapsed between a past and present event [53]. Radvansky and Zacks [54] further explain how spatiotemporal location is a major organizing factor in memory for events and serves as the basis of an event model — or a multidimensional representation of a real-world event. For example, event models can be dependent on a particular perspective in which the information was learned, such as experiencing a space from a birds-eye view or a first person perspective [54]. It is clear how this can be problematic when asking students to consider a timeline prior to Earth's existence or 
a time after Earth has formed, but especially from a location requiring a space-based perspective as these two perspectives in time and space cannot be experienced directly.

Recent research in astronomy education is attempting to remedy the disconnect between spatiotemporal location by exploring spatial thinking through students' views of Earthbased and space-based frames of reference. Plummer and colleagues $[47,55,56]$ have investigated children's perceptions of the daily apparent motion of the Sun, Moon, and stars. Plummer [56] found that children often explained that the Sun moved across the sky rather than Earth rotating, which was also similar to studies by Vosniadou and colleagues. However, it was observed that even children that could explain the Sun's apparent motion correctly often could not apply the knowledge of Earth's rotation to account for the motion of the stars. The researchers concluded, "knowing the scientific description of both an Earth-based phenomenon and how objects move in space does not necessarily lead to an understanding why motion in one frame of reference causes the appearance of motion in the other" [56].

Others have explored college students' spatial reasoning abilities. Black [57] found a possible relationship between poor spatial abilities - most specifically mental rotationand earth science misconceptions. Similarly, Heyer et al. [58] measured spatial thinking abilities in undergraduate nonscience majors and found results which suggested the relationship between spatial reasoning and astronomical ability can explain roughly $25 \%$ of variation in student achievement. Thus, fostering spatial reasoning skills not only in children, but in traditional college astronomy courses, may help to improve conceptual difficulties related to Earth's location in time and space.

Existing work on spatial thinking in the science education community may provide a meaningful pathway toward incorporating more research and instruction on spatiotemporal location. Spatial reasoning skills have previously predicted achievement in science, technology, engineering, and mathematics (STEM) fields; however, students are infrequently taught these skills or provided opportunities to practice honing these skills in the classroom [59]. The likelihood that children and college-aged students will construct scientifically accurate astronomical explanations of Earth's place in time and space in the Universe requires the spatial skills of mental rotation, spatial perception, and spatial visualization $[45,57,58,60]$; in other words, students must possess or learn the ability to extrapolate in three dimensions. Eriksson et al. [61] explored students ability to discern the multidimensional structure of the Universe and found this ability was relatively rare for undergraduate students. The researchers highlight how improving knowledge and awareness on relative sizes and distances in the Universe are limited to only two dimensions.

The ability to perceive the Universe in multiple dimensions calls for more than is provided in traditional disciplinary knowledge and requires the use of hands-on experiences, computer simulations, and other virtual tools to enhance astronomical learning [61]. Researchers continue to use visual representations of the Universe in new and different ways to help students grasp the 3D structure and hierarchy of the Universe. For example, Schneps et al. [62] provided high school students with virtual astronomy simulations on iPads to see if immersive, hands-on technology could help students to learn spatial scales. Students engaged in brief interactions with 3D simulations with a pinch-to-zoom interface, and these simulations helped students to develop spatial skills with minimal instructional support [62]. Furthermore, as a precursor to our work, L. S. Brock developed the framework for a museum exhibition that connected spatial and temporal scales as part of a master's thesis [63]. The exhibition centered on a "walking timeline" of Earth's climatological, biological, and geological history in which visitors would view Earth from a space-based perspective at different distances, and thus, points in time throughout the history of the Universe.

It should be cautioned, however, that technology alone does not rectify students' difficulties with spatial reasoning. Urban and colleagues warn that without providing an opportunity to experience motions of parallax, for example, students may not be successful when attempting to extrapolate from $2 \mathrm{D}$ to $3 \mathrm{D}$ representations of the Universe, even with the use of computer simulations [61]. Schneps et al. [62] reported that unstructured 3D simulations did not help students overcome their naive ideas about the seasons, and the presence of misconceptions would require scaffolding specifically designed to address the implications of such naive ideas. Mental rotation, such as a spatial transformation of an object, and the ability to take in a scene from a different viewpoint (i.e., perspective taking) are simultaneously required in astronomy courses. Incorporating new technologies into the astronomy classroom is a useful tool for developing spatial awareness via mental rotation, but these tools must be accompanied by effective teaching strategies to aid students in constructing accurate scientific representations of the Universe.

Hegarty and Waller [64] highlight a dissociation between spatial abilities that require both mental rotation and the ability to take on a different visual perspective that might explain why spatial reasoning difficulties often occur in astronomy. This work provides insight on how we can improve on this in our new research agenda. In experiments on perspective taking in large-scale spatial situations, Hegarty and Waller found a correlation with being in a memorized environment and people's self-reported sense of direction. In other words, the more familiar a person is with their environment, the more likely a person is able to succeed at large-scale spatial cognition tasks (e.g., planning or navigating a route), which requires the individual to imagine themselves in a particular place or orientation and determine the direction of travel from one location to 
another in the given environment. Surprisingly, successful planning or navigation was shown to be true for perspective taking whether the configuration was viewed or imagined, which is promising for our agenda relating Earth's place in time and space in the Universe.

Approaches toward developing students' abilities to construct representations of Earth's place in time and space will require attention to both mental rotation and perspective taking. Plummer and colleagues have dedicated significant efforts with perspective taking that connect the view of Earth from the Sun in both Earth- and space-based reference frames [55,65]. For example, students must be able to comprehend why our Earth-based perspective of the Sun's motion around Earth is opposite in a space-based reference frame. Our work will require an extension to perspective taking that incorporates Earth's location in both time and space in the Universe. Adding the fourth dimension of time increases the complexity of mental rotation and perspective taking, but coupling temporal and spatial awarenesses can be used to introduce students to different points in time throughout cosmological history. Time often influences how an event is perceived and stored into memory [54], such as the case when students conflate Earth forming at the same time as the Universe, which suggests students' temporal perspectives influence the relative orientation and hierarchy of astronomical events in the past, present, and future. We believe it is worthwhile to investigate perspective taking that incorporates a temporal point of view.

\section{Geological time}

In this section, we discuss contributions that have addressed geological time. It should first be noted that there is some disparity regarding the definition of the term geological time within the literature. Looking ahead, we use geological time to refer to the time period from Earth's formation to the present day, encompassing all of Earth's 4.54 billion year geological history. We use cosmological time to refer to the much larger span of time stretching back approximately 13.8 billion years (to the age of the known Universe) to present day. Some researchers use geological time interchangeably with the term deep time, though the beginning point in time varies from either the origin of the Universe or the formation of Earth. Clearly, a rather vast span of time exists between these two origin events. This nomenclature may not have implications for researchers who focus on a starting point and proceed to investigate students' knowledge of time restricted to post-Earth formation and related events; however, it is important to clarify terminology for future studies attempting to incorporate students' understanding of cosmological time into the existing literature. For clarity, we refrain from using the term deep time in this paper but feel it most appropriately encompasses both cosmological and geological timescales.
Studies exploring geological time have been both qualitative and quantitative, asking subjects to respond to open-ended questions, recall certain events or dates, select an appropriate age range, or organize events or dates in chronological order. Studies of this nature help to gather information regarding learners' knowledge of absolute ages and their relative relationships within a time frame. A great deal of research on geological time identifies that learners struggle to provide the age of Earth as well as place Earth events from formation to present day in appropriate timescales within a timeline.

Work from Trend [32] determined that students, pre-, and in-service teachers developed their own personal "deep time frameworks," or an individual chronological order of key events and dates, which greatly differed from a scientific acceptance. Data show subjects across all studies and age groups have difficulty providing scientifically accurate approximations of time, favoring instead qualitative terms (e.g., ancient, less ancient, recent) $[30,32,66]$. Similarly, a more recent geological ordering study by Libarkin et al. [34] showed how few students were able to create timelines that were scientifically acceptable and relative spacing of events by students was nonscientific. When asked about Earth's age, Marques and Thompson [67] found younger students typically provided estimates of hundreds or thousands of years, whereas older students typically responded with millions, billions, or sometimes even trillions. Libarkin and Anderson [35] conducted a qualitative analysis of students enrolled in introductory science courses at university and concluded that fewer than $50 \%$ of all students in the study believed that Earth was 4-5 billion years old, and at some institutions, this was less than $10 \%$. These findings are important for our work because for our agenda what will be needed is to figure out how these time scales relate to a much larger framing of cosmological time.

Studies of students' knowledge of how time functions in an evolutionary biology context are related to geological time, and knowledge of these individual topics has been shown to impact each other. For example, Cotner et al. [68] demonstrated that students' beliefs of the age of Earth impacted their ability to accept evolution. If one does not believe or understand that Earth is billions of years old, the same disparity could be applied to the subsequent age of life and its evolution on Earth. This can be seen in other studies as well. Interdisciplinary research on geology and evolutionary biology has investigated students' knowledge of the first appearance of various life forms on Earth (e.g., dinosaurs). Early work with students in secondary school demonstrated how life is often perceived as arising concurrently with the formation of Earth [67]. Delaughter and Stein [33] also found that approximately $25 \%$ of students in their study gave answers to time-related questions in which dinosaurs died long before life even began on Earth. Other students believed Earth was younger than life, and many 
students placed the beginning of life at less than 1 million years. Collective findings reveal a disconnect between the relative relationships of the age of Earth, the time and characteristics of life's first appearance on Earth, and the subsequent evolution of life on Earth [23,33,36,67].

Another interdisciplinary study by Catley and Novick [23] evaluated perceptions of geological time by assessing college students' knowledge of seven key macroevolutionary events related to life on Earth (e.g., first appearance of prokaryotic life, eukaryotic life, dinosaurs, hominid lineage). Results showed low levels of accuracy regarding order of magnitudes among students, extreme variability in estimates, and a tendency to estimate dates closer to the present time. Another interesting finding from this study was students with a greater biology background, even those who had taken a prior course on evolution, did not fare better on their knowledge of geological time. A similar trend was observed by Libarkin and colleagues [34], in which the researchers noticed that students had less difficulty placing events in the correct order but had a poor understanding of the scale of time between evolutionary events.

Not all research related to geological time has been based on events. A handful of studies have focused on students' underlying cognitive bases for understanding geological time itself rather than students' abilities to recall specific events or dates [69]. Ault [29] examined elementary students' understanding of time from a psychological perspective, influenced by Piaget's very early research on time cognition that related children's understanding of time to motion [3]. Ault argued that young children's conceptions of conventional time were not an impediment towards an understanding of the geological past. However, Dodick and Orion [70] found Ault's results to be inconsistent with their own work and argued children's ability to grasp geological time cannot be related to an ability to grasp conventional timescales as the timescales required for geological time are much greater. This point is especially important for our work, considering cosmological timescales are much greater than geological scales of time.

Dodick and Orion [24,31,69-71] have dedicated significant research efforts exploring how students' perceive geological time cognitively. Their studies with Israeli middle and high school students led to the development of the Geological Time Aptitude Test (GeoTAT) [69] and an accompanying theoretical framework for exploring the comprehension required for the enormous scale of geological time. To develop this theoretical framework, Dodick and Orion [70] utilized and adapted Montangero's model of diachronic thinking [72] to examine how students reconstructed geological transformations over time. Three schemas were focused on for the study: transformation, temporal organization, and interstage linkage. Transformation relies on qualitative or quantitative change, temporal organization defines sequential orders of events or processes, and interstage linkage serves as the connection between key phenomena. The researchers determined that transformation seems to influence the other two themes and was dependent upon an ability to make connections from past events to present events. This work further connected the relationship between temporal and spatial thinking, particularly across the geological timescale. Dodick and Orion [69] focused on the logical strategies students used when faced with temporal problems and what factors might interfere with the process, notably students' prior geological knowledge and spatial visualization. Cheek's later work [73,74] identified how large numbers can place limits on a student's ability to understand associated geological processes. However, she found that students had little difficulty dealing with sequencing a set of geological events in conventional time and argues geological content knowledge is likely a larger factor to consider.

Synthesizing across the geological time literature, students generally approach large orders of magnitude in time from a qualitative perspective, resorting to grouping or sequencing to make sense of the age and duration of events. It is clear the quantitative aspect of large numbers that is required for learners to grasp geological events can be problematic. Landy et al. [75] investigate how difficulty with large numbers interferes with an understanding of concepts that require their use. Their work shows that people tend to treat thousands, millions, billions, and trillions as a uniformly spaced list in magnitude on a number line. The outcome is that any associated scientific events that occur on timescales using such numbers become misrepresented. Lee et al. [21] also found that students tended to overestimate durations of time for large temporalmagnitude categories; however, students also tended to overestimate durations of time smaller than one millisecond. In related work, Tretter et al. [76] explored students' conceptions of spatial scales and noticed students had difficulty providing accurate sizes of objects at the microscopic scale. These works suggest both temporal and spatial magnitude scales outside of the human experience rangeboth large and small — can pose challenges for learners.

Arguably, this suggests an approach toward improving students' grasp of the geological timescale and its relationship to cosmological time might require initially removing the quantitative aspect of time, instead focusing on the connection between past and present events. By making the sequence and order of events that occur on large timescales more concrete, large scales of time can become a more approachable concept for students. Furthermore, providing relevant, qualitative points of interest in time-such as the fact there is more time between Tyrannosaurus rex and Stegosaurus than human's entire existence on Earth-is perhaps far more profound and relatable to students than a memorization of dates on a timeline.

\section{Cosmological time}

The relationship between cosmological and geological timescales is not only important for our research agenda, 
but there are many parallels between the cognitive synthesis required to understand both of these immense timescales. Our previous discussion on prior research in geological time shows little connection to research on Earth's formation and, similarly, we will see how research on cosmological time does not connect to events post-Earth formation. The connection between the cosmological timeline, astronomical events surrounding Earth's formation, and the beginning of the geological timescale to the subsequent events that occurred after our planet formed must be strengthened in order for students to construct a clear representation of Earth's place in space and time in the Universe. By excluding this connection, not only does this mathematically exclude a significant portion of known time, but this also fails to capture the complex and important relationship between Earth's formation and the rest of the cosmic landscape.

The formation of Earth and its subsequent evolution is arguably at the transition between astronomy and geology, though few geologically focused studies tend to include a cosmic event. Trend's [32] study is an example as it included geological events and the big bang; however, this study was focused more on Earth's history and did not provide enough information for a comprehensive analysis of how students' view time throughout cosmic evolution in an astronomical context. Another more recent study by Delgado [11] incorporated the big bang and the age of Earth. Unlike Trend's study, Delgado was able to show that college-aged students in a history course could successfully provide the approximate time estimate for two astronomical events - the big bang and the age of Earth. The big bang is a popular choice to include in studies, but this appears as more of a starting marker for the timeline rather than a key event to explore more in-depth in the study. Furthermore, this excludes other key astronomical events and spatial or temporal scales that bridge the gap from big bang to Earth's formation, which need to be incorporated into research and class agendas. Although Delgado's study did not include the addition of other astronomical events, his work does show students are capable of learning about cosmological timescales when they are given adequate instructional time.

Related work regarding the big bang and our Universe, though not directly focused on cosmological time, has shown that students possess a variety of nonscientific ideas about the origin of the Universe that likely influence students' conceptions of time or vice versa. In a study exploring nearly 1000 students' ideas about the big bang from middle school through college, Prather et al. [13] found that $70 \%$ of students responded that matter existed prior to the big bang. In this study as well as others $[4,10,12]$, it is commonly observed that students describe the big bang as a process that formed Earth or our Solar System in a variety of different ways. Unfortunately, students even self-report taking an astronomy course(s) in the past and proceed to incorrectly respond to questions.
These studies did not investigate conceptions of time per se and were more focused on astronomical content knowledge; however, it is important to note how improved connection between the duration and occurrence of such astronomical events could strengthen an understanding of the events themselves.

It could be incorrectly assumed that students do not know much about the origin of our Universe or how our Solar System formed at all. However, looking deeper at exactly how students respond to these types of questions, it appears that many students $d o$ possess adequate building blocks to reach a more scientific understanding with proper, targeted instruction. One way to improve students' fragmented and incorrect knowledge is through the use of learning progressions. Plummer et al. [12] developed a hypothetical learning progression as a first step that could lead students to explain the formation of our Solar System through a big idea approach. A big idea approach connects multiple scientific concepts together and would inherently be connected to an understanding of cosmological time. In other words, this learning strategy provides a map of the fundamental astronomical and physical concepts involved in the planetary formation process - such as gravitational cloud collapse and angular momentum in the disk-that can guide students and teachers of different levels and backgrounds throughout the learning process, focusing on the big ideas of the topics rather than specific details. This is a new approach for astronomy courses, and explicitly incorporating components of cosmological time into learning progressions may help to tie big ideas in astronomy together in the future.

\section{Earth's place in the Universe}

What is lacking in the astronomy education community is a connection between temporal and spatial scales as related to a larger, comprehensive view of Earth's place in time and space in the Universe. Time refers to cosmic events that occurred before and after Earth's formation, whereas place here not only refers to Earth's physical location in the Universe (i.e., Earth orbits the Sun in the Solar System, which is part of the Milky Way Galaxy, Local Group, and Local Supercluster) but also the significance of Earth and humanity in this new era of exoplanet detection and discovery. Use of the phrase Earth's place in the Universe in other contexts has referred to Earth-centric topics that occur in our own Solar System, mainly focusing on Earth-Moon-Sun motions, which has been listed in previous [26] and current [27] educational standards in the United States.

A multidisciplinary temporal and spatial approach to Earth's place in time and space in the Universe is not necessarily a new idea in the domain of natural history, however. A few historical researchers have been exploring how humanity fits into the larger context of the Universe for the past few decades. This concept has more recently 
become known as the field of big history [77] and has subsequently emerged outside of history's disciplinary borders. The Big History Project movement has spawned the development of big history courses in secondary and postsecondary education, with these courses covering topics from parallax to megafauna to the Industrial Revolution [11,78]. Big history courses comply with the National World History Standards [79] and, in certain high schools, have even replaced required World History courses.

Though the approach is broad and encompasses the entire spectrum of history from the origin of the Universe to the present day, the focus is largely centered on placing human history into the scale of cosmic history and not necessarily about understanding the context of cosmological events from a scientific perspective. In turn, some cosmologists have argued for a more objective approach to big history and have developed their own empirical approach, coining it cosmic evolution [80-82]. Chaisson [80] argues that big historians approach cosmic evolution from an anthropocentric perspective that suggests the Universe "arose specifically to produce us." Although the scientific content in big history courses may be arguably less than needed for a scientifically focused course, the design of big history is a step in the right direction with regards to interdisciplinary learning and connecting students - in some way-to the seemingly irrelevant history of the Universe. What remains to be explored is how students, particularly nonscience majors, approach this idea of Earth's place in the Universe and how these students' beliefs interact with the acquisition of astronomical knowledge. Research conducted on students' perspectives of Earth relative to the cosmic landscape provides an excellent pathway for future investigations of students' grasp of temporal and spatial scales in astronomy.

\section{E. Current research}

New research strategies that target curricula and instruction related to Earth's place in time and space in the Universe are essential in order to improve how students construct temporal knowledge in an astronomical context. Topics such as astrobiology and the ongoing search for and study of exoplanets already exist in the astronomical realm and provide excellent pathways for incorporating education related to temporal and spatial scales. Astrobiology inherently includes the large temporal scales required to describe how life first arose and evolved on Earth nearly 4 billion years ago, as well as discussion of spatial scales at the microscopic level in order to become familiar with Earth's first life at the cellular level. Furthermore, both astrobiology and exoplanetary science require a grasp of the large orders of magnitude in distance to bodies in our Solar System and beyond.

From previous literature and our own research efforts, we believe targeted instruction related to time serves as a missing link toward improving astronomy education and students' conceptions of Earth's relationship to the Universe.
We have synthesized time-related research across multiple disciplines to inform the direction of a new, needed research agenda in astronomy. The first author's work over the past three years has focused specifically on undergraduate students' knowledge of the relationship between cosmological and geological time and causality related to astronomical phenomena (e.g., what had to exist before Earth and the Solar System could form). Our continued, collaborative work on time exploring the aforementioned concepts in introductory astronomy courses has enabled this new research agenda to come to fruition. We are developing means to uncover students' perceptions on time, such as through the development of a timeline tool that will be used to gauge students' knowledge of key macroevolutionary events across time from the origin of the Universe to the present day.

Furthermore, we have been piloting open-ended questions with undergraduate students that explore how underlying epistemological and ontological conceptions influence learning in introductory astronomy courses. In our research, we looked at 170 undergraduates' responses to 1 of 4 questions related to (i) when Earth formed with respect to the Universe, (ii) what astronomical events had to occur, and (iii) what materials needed to exist before Earth formed, and (iv) if all planets in the Universe formed at the same time as Earth. Iterative analysis of responses confirmed prior researchstudents have little knowledge of absolute and relative timescales and a variety of nonscientific ideas about time in astronomy.

However, looking at what students did know in a more positive light provided some interesting insight. Approximately $66 \%$ of students responding to question (i) knew Earth formed after the Universe and 60\% of students said the big bang happened before Earth formed; although students still did not provide responses that demonstrated a numerically accurate representation of the timescales involved. Examining question (iii), students were quite unclear about the materials that existed prior to Earth's formation and often became bogged down by vocabulary terms. Some students provided the names of elements in their responses, though only $33 \%$ identified the existence of hydrogen, $7 \%$ helium, and $23 \%$ oxygen prior to Earth's formation. Other responses involving dust, matter, and atoms to dirt or even life were also provided as Earth precursors. Finally, a surprising $72 \%$ correctly stated for question (iv) that all planets in the Universe did not form at the same time, but the majority could not really explain why it was true.

The above results highlight the potential power of time-related knowledge in astronomy and further support the notion that interdisciplinary instruction connecting astronomy with chemistry, geology, and physics in the classroom is worthy of more research. Many students even at the college level with prior astronomy coursework appear to be unclear how Earth fits into the cosmic 
landscape. This content area has largely remained a focus in the literature on younger age groups, but our research suggests explorations of student perceptions on Earth's place in time and space in the Universe may be pertinent to investigate for older populations of students and is worthy of more research as well. Despite general difficulties with concepts related to Earth's place in time and space in the Universe, students were clearly able to activate the resources they possessed in the context of questions asked. Introducing Hammer's notion of resource framing into classrooms may help students highlight their existing astronomical knowledge [20], and explicitly dedicating classroom time to learning about new space-based frames of reference and nongeocentric perspectives of time may provide means for students to continue to build upon their existing knowledge resources.

\section{TIME IN THE FUTURE: RECOMMENDATIONS FOR RESEARCH AND INSTRUCTION}

Extrapolating data from studies on geological time provides reasonable evidence to support the fact that students of all ages, teachers, as well as the general public struggle to grasp the span of immense timescales - such as Earth's age or the age of the Universe-in both absolute and relative timescales. Catley and Novick [23] eloquently highlighted some of the common overarching complications observed from temporal reasoning: (i) subjects provided time frames that spanned unimaginably large periods of time (e.g., trillions), (ii) the ability to discriminate between very large numbers was rare (e.g., millions vs billions), (iii) the frequency of time estimates to the correct order of magnitude was rare, and (iv) it was a common misconception among school children and college students that life arose concurrently with the formation of Earth. These findings are easily extended to time in astronomy from our work and recent literature, with both children and college students: (i) defining the origin event that formed the Universe as an event that formed Earth and/or our Solar System, (ii) underestimating and overestimating the age of the Universe by millions or billions of years, and (iii) describing — at whatever time the Universe formed - it as an event organizing preexisting matter.

Timescales of this size are not easy to comprehend or relate to admittedly, and are far outside the range of human experience as Landy et al. [75] point out, which may explain why the majority of people fail to provide sufficient knowledge of these scales and their associated events. Yet, the ability to construct scientifically accurate temporal frameworks remains necessary for learning about and truly understanding astronomy at its core, and effectively building these knowledge structures may help students to grasp astronomical concepts more concretely. The question remains: How can we as educators and instructors improve instruction related to time?
Suggestions from the literature have been provided from all facets of Earth and space science community. In the geosciences, Dodick and colleagues suggest that learning materials and tasks related to time should be organized, readable, and relevant, so that learners can make sense of the learning task. They argue it is important that the abstract essence of geological time with its large numerical values can be made relevant to students if it is anchored to visualizable events and not just a quantitative understanding. In evolutionary biology, Catley and Novick [23] used an instructional strategy that also incorporated visualizations of large evolutionary events through the use of phylogenetic trees, or trees which showed evolutionary relationships. They explain these trees serve as markers that may help incorporate instruction on spacing and make it relatable to evolutionary time. Similarly, Delgado [11] suggested time landmarks to function as pinpoints across learning disciplines so students can orient themselves to specific points in time. Conversely, Trend [32] said timelines may be appropriate for historical events but not for cosmological and geological time, as the scale cannot be conceptualized onto a single timeline if ongoing and consistent change is to be expressed.

Unlike in geology and evolutionary biology, time in its cosmological sense is indeed much more vast than the span of all we know here on Earth and its relatively young age in comparison. Cataloging and sequencing astronomical phenomena into simple, orderly events is less than ideal considering the formation and destruction of stars, planets, and galaxies is recurring even as you read this. We encourage future work to focus on causality, connection across major astronomical events, and relevancy to students' personal lives rather than memorization of ages, dates, or a list of events. We have synthesized the following take-away guidelines as recommendations for future research and instruction related to time at all levels:

- Explicit instruction through visualizations and selfguided active learning strategies that explore the relationship between cosmological and geological spatiotemporal scales (i.e., the age of the Universe versus the age of Earth; the sizes of Earth and the Solar System versus the sizes of galaxies and the Universe) and how the age of Earth and the Universe were determined.

- Incorporation and clarification of cosmic events into the classroom including the big bang and after, leading up to Earth's formation (e.g., cosmic microwave background, formation of first stars).

- Instruction on the composition of the Universe and visualizations of the large-scale hierarchical structure of planets, stars, solar systems, and galaxies.

- Explicit instruction with clarification on the size of astronomical objects relative to the size of the Universe.

- Increased instructional attention on processes of stellar nucleosynthesis including where, when, and 
how the elements on the periodic table arose and how this is related to astronomy and Earth's composition.

- Increased attention on the interdisciplinary knowledge related to physics, particularly gravity and its influence on processes across large spatial scales (e.g., expansion of the Universe, planet formation) and how this is related to time.

Developing effective curricula and instruction that produces temporally literate learners in astronomy further helps students to construct accurate scientific representations of astronomical phenomena. The strides made over the past few decades in astronomy education research have been incredibly valuable and paramount in shaping how introductory astronomy is taught. However, the available literature shows more can be accomplished with temporally related instruction and interdisciplinary knowledge in astronomy - for both instructors and their students [4,5]. More recent research has begun to tackle these ideas related to temporal instruction and interdisciplinary knowledge, though little discussion on Earth's place in time and space in the Universe has been investigated. Efforts to improve how learners construct temporal frameworks span far beyond the discipline of astronomy itself and are dependent upon interdisciplinary and multidisciplinary cooperation and instruction through a collaborative community effort across Earth and space sciences. Those in the astronomy and physics education communities, as well as others in the broad community of the physical sciences, are encouraged to pursue study related to our new research agenda described in this paper. We appreciate the incorporation of time-related research and instruction into teaching and look forward to reports on successful methodologies that investigate temporal perceptions as related to the larger picture of Earth's place in time and space in the Universe.

\section{ACKNOWLEDGEMENTS}

We would like to acknowledge support from the Howard Hughes Medical Institute through Grant No. 4215580. We also would like to thank Tim Swindle and the Lunar and Planetary Laboratory for the generous contributions which enabled this article to be published.
[1] J. Piaget, The Child's Perception of the World (Kegan Paul, London, England, 1929).

[2] J. Piaget and M. Gabain, The Child's Conception of Physical Causality, in International Library of Psychology, Philosophy, and Scientific Method (Kegan Paul, London, England, 1930), p. vii.

[3] J. Piaget, The Child's Conception of Time (Ballantine Books, New York, 1969).

[4] J. M. Bailey and T.F. Slater, A review of astronomy education research, Astron. Educ. Rev. 2, 20 (2003).

[5] A. Lelliott and M. Rollnick, Big ideas: A review of astronomy education research 1974-2008, Int. J. Sci. Educ. 32, 1771 (2010).

[6] T. Slater, What topics are taught in introductory astronomy courses?, Phys. Teach. 39, 52 (2001).

[7] J. Fergusson, C. Oliver, and M. R. Walter, Astrobiology outreach and the nature of science: the role of creativity, Astrobiology 12, 1143 (2012).

[8] C. Oliver and J. Fergusson, Astrobiology: A pathway to adult science literacy?, Acta Astronaut. 61, 716 (2007).

[9] J. T. Staley, Astrobiology, the transcendent science: The promise of astrobiology as an integrative approach for science and engineering education and research, Curr. Opin. Biotechnol. 14, 347 (2003).

[10] C. Wallace, E. Prather, and D. Duncan, A study of general education astronomy students' understandings of cosmology. Part IV, Astron. Educ. Rev. 11, 010104 (2012).

[11] C. Delgado, Navigating deep time: Landmarks for time from the big bang to the present, J. Geosci. Educ. 61, 103 (2013).
[12] J. D. Plummer, C. Palma, A. Flarend, K. Rubin, Y. S. Ong, B. Botzer, S. McDonald, and T. Furman, Development of a learning progression for the formation of the solar system, Int. J. Sci. Educ. 37, 1381 (2015).

[13] E. E. Prather, T. F. Slater, and E. G. Offerdahl, Hints of a fundamental misconception in cosmology, Astron. Educ. Rev. 1, 28 (2002).

[14] How People Learn: Brain, Mind, Experience, and School, edited by J. D. Bransford, A. L. Brown, and R. R. Cocking (National Academy Press, Washington, DC, 2004).

[15] Mental Models, edited by D. Gentner and A. L. Stevens (Erlbaum, Hillsdale, NJ, 1983).

[16] S. Vosniadou and W.F. Brewer, Mental models of the earth: A study of conceptual change in childhood, Cogn. Psychol. 24, 535 (1992).

[17] Mental Models, edited by P. N. Johnson-Laird (Harvard University Press, Cambridge, MA, 1983).

[18] A. A. DiSessa, Knowledge in Pieces, edited by G. Forman and P. B. Pufall (Erlbaum, Hillsdale, NJ, 1988), pp. 49-70.

[19] D. Hammer, Misconceptions or P-Prims: How may alternative perspectives of cognitive structure influence instructional perceptions and intentions, J. Learn. Sci. 5, 97 (1996).

[20] D. Hammer, A. Elby, R. Scherr, and E. F. Redish, in Transfer of Learning from a Modern Multidisciplinary Perspective, edited by J.P. Mestre (Information Age Publishing, Greenwich, CT, 2005), pp. 89-119.

[21] H. Lee, O. L. Liu, C. A. Price, and A. L. M. Kendall, College students' temporal-magnitude recognition ability associated with durations of scientific changes, J. Res. Sci. Teach. 48, 317 (2011). 
[22] C. Cervato and R. Frodeman, The significance of geologic time: Cultural, educational, and economic frameworks, Geol. Soc. Am. Bull. 2486, 19 (2012).

[23] K. M. Catley and L. R. Novick, Digging deep: Exploring college students' knowledge of macroevolutionary time, J. Res. Sci. Teach. 46, 311 (2009).

[24] J. Dodick and N. Orion, Geology as an historical science: Its perception within science and the education system, Science \& Education 12, 197 (2003).

[25] Advancement of Science, Tech. Rep. (American Association for the Advancement of Science, New York, NY, 1993), p. 2061.

[26] Committee on Science Learning, H. A. Schweingruber, R. A. Duschl, and A. W. Shouse, Taking Science to School: Learning and Teaching Science in Grades $K-8$ ( National Academies Press, Washington, DC, 2007), p. 404.

[27] N. L. States, Next Generation Science Standards: For States, By States (National Academies Press, Washington, DC, 2013).

[28] T. College Board, College Board Standards for College Success (College Board, New York, NY, 2009), p. 242.

[29] C. R. Ault, Time in geological explanations as perceived by elementary-school students, J. Geol. Educ. 30, 304 (1982).

[30] R. Trend, An investigation into understanding of geological time among 10- and 11-year-old children, Int. J. Sci. Educ. 20, 973 (1998).

[31] J. Dodick, Understanding evolutionary change within the framework of geological time, J. Educ. 42, 245 (2007).

[32] R. Trend, Deep time framework: A preliminary study of U.K. primary teachers' conceptions of geological time and perceptions of geoscience, J. Res. Sci. Teach. 38, 191 (2001).

[33] J. E. Delaughter and S. Stein, Preconceptions about earth science among students in an introductory course, EOS 79, 429 (1998).

[34] J. C. Libarkin, J. P. Kurdziel, and S. W. Anderson, College student conceptions of geological time and the disconnect between ordering and scale, J. Geosci. Educ. 55, 413 (2007).

[35] J. C. Libarkin and S. W. Anderson, Assessment of learning in entry-level geoscience courses: Results from the geoscience concept inventory, J. Geosci. Educ. 53, 394 (2005).

[36] J. C. Libarkin, S. W. Anderson, J. Dahl, M. Beilfuss, and W. Boone, Qualitative analysis of college students' ideas about the earth: Interviews and open-ended questionnaires, J. Geosci. Educ. 53, 17 (2005).

[37] K. J. Schoon, Students' alternative conceptions of earth and space, J. Geol. Educ. 40, 209 (1992).

[38] S. Vosniadou and W.F. Brewer, Mental models of the day/night cycle, Cogn. Sci. 18, 123 (1994).

[39] J. Nussbaum and J. D. Novak, An assessment of children's concepts of the earth utilizing structured interviews, Sci. Educ. 60, 535 (1976).

[40] J. Nussbaum, Children's conceptions of the earth as a cosmic body: A cross age study, Sci. Educ. 63, 83 (1979).

[41] G. Özdemir and D. B. Clark, An overview of conceptual change, Eurasia J. Math. Sci. Technol. Educ. 3, 351 (2007).

[42] D. Gentner, Spatial Schemas in Abstract Thought (MIT Press, Cambridge, MA, 2001), pp. 203-222.
[43] M. Straatemeier, H. L. J. van der Maas, and B. R. J. Jansen, Children's knowledge of the earth: A new methodological and statistical approach, J. Exp. Child Psychol. 100, 276 (2008).

[44] T. Hannust and E. Kikas, Young children's acquisition of knowledge about the Earth: A longitudinal study, J. Exp. Child Psychol. 107, 164 (2010).

[45] E. Kikas, The effect of verbal and viso-spatial abilities on the development of knowledge of the Earth, Res. Sci. Educ. 36, 269 (2006).

[46] J. D. Plummer, A cross-age study of children's knowledge of apparent celestial motion, Int. J. Sci. Educ. 31, 1571 (2009).

[47] J. D. Plummer, Spatial thinking as the dimension of progress in an astronomy learning progression, Studies Sci. Educ. 50, 1 (2014).

[48] E. G. Chrysikou and C. H. Ramey, Factors influencing the adoption of temporal metaphors, Proc. of the Annual Meeting of the Cognitive Sci. Soc. 29, 1724 (2007).

[49] T. Matlock, M. Ramscar, and L. Boroditsky, On the experiential link between spatial and temporal language, Cogn. Sci. 29, 655 (2005).

[50] I. Levin, The development of the concept of time in children: An integrative model, in Time, Action and Cognition: Towards Bridging the Gap, edited by F. Macar, V. Pouthas, and W. J. Friedman (Springer, Netherlands, Dordrecht, 1992), pp. 13-32.

[51] L. Boroditsky, Metaphoric structuring: Understanding time through spatial metaphors, Cognition 75, 1 (2000).

[52] C. Kurby and J. Zacks, Age differences in the perception of hierarchical structure in events, Memory \& Cognition 39, 75 (2011).

[53] S. Grondin, Timing and time perception: A review of recent behavioral and neuroscience findings and theoretical directions, Attention, Perception, and Psychophysics 72, 561 (2010).

[54] G. Radvansky and J. Zacks, Event perception, WIREs Cognitive Science 2, 608 (2011).

[55] J. D. Plummer, C. A. Bower, and L. S. Liben, The role of perspective taking in how children connect reference frames when explaining astronomical phenomena, Int. J. Sci. Educ. 38, 345 (2016).

[56] J. D. Plummer, Children learning to explain daily celestial motion: Understanding astronomy across moving frames of reference, Int. J. Sci. Educ. 33, 2181 (2011).

[57] A. A. Black, Spatial ability and earth science conceptual understanding, RELEA 16, 45 (2005).

[58] I. Heyer, S. J. Slater, and T. F. Slater, Establishing the empirical relationship between non-science majoring undergraduate learners' spatial thinking skills and their conceptual astronomy knowledge, RELEA 16, 45 (2013).

[59] D. H. Uttal, N. G. Meadow, E. Tipton, L. L. Hand, A. R. Alden, C. Warren, and N. S. Newcombe, The malleability of spatial skills: A meta-analysis of training studies, Psychol. Bull. 139, 352 (2012).

[60] J. Wilhelm, Gender differences in lunar-related scientific and mathematical understandings, Int. J. Sci. Educ. 31, 2105 (2009).

[61] U. Eriksson, C. Linder, J. Airey, and A. Redfors, Who needs 3D when the universe is flat?, Sci. Educ. 98, 412 (2014). 
[62] M. H. Schneps, J. Ruel, G. Sonnert, M. Dussault, M. Griffin, and P. M. Sadler, Conceptualizing astronomical scale: Virtual simulations on handheld tablet computers reverse misconceptions, Comput. Educ. 70, 269 (2014).

[63] L.S. Brock, To the Universe and Back: Increasing Earth and Space Science Literacy Through a Deep Time Exhibition, Master's thesis, Northern Arizona University, 2015.

[64] M. Hegarty and D. Waller, A dissociation between mental rotation and perspective-taking spatial abilities, Intelligence 32, 175 (2004).

[65] L.S. Liben and R. M. Downs, Understanding personspace-map relations: Cartographic and developmental perspectives, Dev. Psychol. 29, 739 (1993).

[66] R. Trend, An investigation into the understanding of geological time among 17-year-old students, with implications for the subject matter knowledge of future teachers, Int. Res. Geographical Environ. Educ. 10, 298 (2001).

[67] L. Marques and D. Thompson, Portuguese students' understanding at ages 10-11 and 14-15 of the origin and nature of the Earth and the development of life, Res. Sci. Technol. Educ. 15, 29 (1997).

[68] S. Cotner, D. C. Brooks, and R. Moore, Is the age of the earth one of our "sorest troubles?" Students' perceptions about deep time affect their acceptance of evolutionary theory, Evolution (Lawrence, Kans.) 64, 858 (2010).

[69] J. Dodick and N. Orion, Cognitive factors affecting student understanding of geologic time, J. Res. Sci. Teach. 40, 415 (2003).

[70] J. Dodick and N. Orion, Measuring student understanding of geological time, Sci. Educ. 87, 708 (2003).

[71] J. Dodick and N. Orion, Building an understanding of geological time: A cognitive synthesis of the 'macro' and 'micro' scales of time, Earth and Mind: How Geologists
Think and Learn about the Earth, edited by C. A. Manduca and D.W. Mogk (Geological Society of America, Colorado, US, 2006), DOI: 10.1130/2006.2413(06).

[72] J. Montangero, The development of temporal inferences and meanings in 5- to 8-year old children, Time, Mind, and Behavior (Springer, Berlin, Heidelberg, 1985), pp. 279-287.

[73] K. A. Cheek, Students' understanding of large numbers as a key factor in their understanding of geologic time, Int. J. Sci. Math. Educ. 10, 1047 (2012).

[74] K. A. Cheek, Exploring the relationship between students' understanding of conventional time and deep (geologic) time, Int. J. Sci. Educ. 35, 1925 (2013).

[75] D. Landy, N. Silbert, and A. Goldin, Estimating large numbers, Cogn. Sci. 37, 775 (2013).

[76] T. Tretter, M. G. Jones, and J. Minogue, Accuracy of scale conceptions in science: Mental maneuverings across many orders of spatial magnitude, J. Res. Sci. Teach. 43, 1061 (2006).

[77] D. Christian, The case for "big history", J. World History 2 , 223 (1991).

[78] D. Christian and W. H. McNeill, Maps of Time: An Introduction to Big History (University of California Press, Berkeley, 2011), Vol. 2.

[79] N. Committee, National Standards for World History: Exploring Paths to the Present: Grades 5-12 (UCLA, Los Angeles, CA, 1994).

[80] E. J. Chaisson, Cosmic evolution-more than big history by another name, in Evolution: A Big History Perspective (Uchitel Publishing House, Volgograd, Russia, 2011), pp. $37-48$.

[81] E. J. Chaisson, Big history's risk and challenge, Expositions 81, 85 (2014).

[82] E. J. Chaisson, The natural science underlying big history, Sci. World J. 2014, 1 (2014). 